\title{
ORACLES FOR DISTANCES AVOIDING A FAILED NODE OR LINK*
}

\author{
CAMIL DEMETRESCU ${ }^{\dagger}$, MIKKEL THORUP ${ }^{\ddagger}$, REZAUL ALAM CHOWDHURY ${ }^{\S}$, AND \\ VIJAYA RAMACHANDRAN $\S$
}

\begin{abstract}
We consider the problem of preprocessing an edge-weighted directed graph $G$ to answer queries that ask for the length and first hop of a shortest path from any given vertex $x$ to any given vertex $y$ avoiding any given vertex or edge. As a natural application, this problem models routing in networks subject to node or link failures. We describe a deterministic oracle with constant query time for this problem that uses $O\left(n^{2} \log n\right)$ space, where $n$ is the number of vertices in $G$. The construction time for our oracle is $O\left(m n^{2}+n^{3} \log n\right)$. However, if one is willing to settle for $\Theta\left(n^{2.5}\right)$ space, we can improve the preprocessing time to $O\left(m n^{1.5}+n^{2.5} \log n\right)$ while maintaining the constant query time. Our algorithms can find the shortest path avoiding a failed node or link in time proportional to the length of the path.
\end{abstract}

Key words. graph algorithms, data structures, shortest paths, network failures

AMS subject classifications. 05C85, 68W01, 68P05, 05C38, 90B18

DOI. $10.1137 /$ S0097539705429847

1. Introduction. In the distance sensitivity problem, we wish to construct a data structure (which we call distance sensitivity oracle) for an edge-weighted directed graph $G$ that supports the following queries:

v-dist $(x, y, v): \quad$ returns the distance from vertex $x$ to vertex $y$ in $G-\{v\}$, i.e., the length (sum of edge weights) of the shortest possible path from $x$ to $y$ in $G$ avoiding vertex $v$, if one exists, and $+\infty$ otherwise.

e-dist $(x, y, u, v)$ : returns the distance from vertex $x$ to vertex $y$ in $G-\{(u, v)\}$, i.e., the length (sum of edge weights) of the shortest possible path from $x$ to $y$ in $G$ avoiding edge $(u, v)$, if one exists, and $+\infty$ otherwise.

We also consider the corresponding path queries, which we denote by $\mathrm{v}$-path $(x, y, v)$ and e-path $(x, y, u, v)$, respectively. In this article, we denote by $n$ the number of vertices and by $m$ the number of edges in $G$. We also assume that edge weights are nonnegative. In our bounds, space is measured as the number of memory words,

* Received by the editors May 22, 2005; accepted for publication (in revised form) July 11, 2007; published electronically January 4, 2008. Part of the results presented in this article for link failures appeared in the Proceedings of the 13th Annual ACM-SIAM Symposium on Discrete Algorithms (SODA'02) and in the Proceedings of the 13th International Symposium on Algorithms and Computation (ISAAC'02).

http://www.siam.org/journals/sicomp/37-5/42984.html

†Dipartimento di Informatica e Sistemistica, Università di Roma "La Sapienza," via Ariosto 25, 00185 Rome, Italy (demetres@dis.uniroma1.it, http://www.dis.uniroma1.it/ demetres). This author's research was supported in part by the Italian Ministry of University and Scientific Research (Project "MAINSTREAM: Algorithms for massive information structures and data streams").

${ }_{\ddagger}^{\ddagger}$ AT\& T Labs-Research, 180 Park Avenue, Florham Park, NJ 07932 (mthorup@research.att.com, http://www.research.att.com/info/mthorup).

${ }^{\S}$ Department of Computer Sciences, The University of Texas at Austin, Austin, TX 78712 (shaikat@cs.utexas.edu, http://www.cs.utexas.edu/ shaikat, vlr@cs.utexas.edu, http://www.cs. utexas.edu/ vlr). The third author's research was supported in part by an MCD Graduate Fellowship and NSF grant CCR-9988160. The fourth author's research was supported in part by Texas Advanced Research Program grant 003658-0029-1999 and NSF grants CCR-9988160 and CCF-0514876.

1299 
where each word can hold the label of a vertex or edge or the weight of an edge or path.

In our directed case, we note that edge failures subsume vertex failures. The reduction is well known from work on disjoint paths. We split each vertex $v$ into an in-vertex $v_{0}$ and an out-vertex $v_{1}$, with an in-out edge $\left(v_{0}, v_{1}\right)$. The original incoming edges are moved to $v_{0}$ and the outgoing edges are moved to $v_{1}$. Now losing the edge $\left(v_{0}, v_{1}\right)$ due to a network failure has the same effect as losing vertex $v$ in the original graph.

1.1. Motivation. Our motivating scenario is a network where node/link failures happen quite rarely. As soon as a node or link failure has been noticed, we want to be able to answer distance queries and provide directions for shortest paths in the network without the failed node or link. On the other hand, we assume that we have plenty of time to compute a new data structure in the background. We model a network as a weighted directed graph where vertices correspond to network nodes, and edges correspond to network links. In this scenario, $\mathrm{v}$-dist $(x, y, v)$ yields the distance from node $x$ to node $y$ in the network avoiding failed node $v$, and e-dist $(x, y, u, v)$ yields the distance from node $x$ to node $y$ in the network avoiding failed link $(u, v)$.

We note that the ability to deal with node/link failures enables us to deal with some other related aspects of the network. For example, by dealing with a link failure $(u, v)$, we actually deal with arbitrary changes to its weight. More precisely, we can simply compute the distance from vertex $x$ to vertex $y$ in the graph where $(u, v)$ has its weight changed to $w$ as $\min \left\{\mathrm{e}\right.$-dist $\left.(x, y, u, v), d_{x u}+w+d_{v y}\right\}$, where $d_{x u}$ is the distance from vertex $x$ to vertex $u$ and $d_{v y}$ is the distance from vertex $v$ to vertex $y$ in the graph. Here, a weight change could model that traffic is moving slower/faster along a certain link. An interesting application of dealing with single weight changes is a local search like the one in [9]. There, one wants to consider a neighborhood of a given weight setting, where each neighbor is obtained by changing a single weight.

Another motivation for solving the distance sensitivity problem arises from recent interest in Vickrey pricing of networks [11, 19]. We describe this application in more detail in section 1.4 .

1.2. Related work. A variant of the distance sensitivity problem, related to reachability in directed acyclic graphs w.r.t. edge failures, was first introduced by King and Sagert in [14], where they consider the problem of supporting sensitivity queries of the kind: "Is there a path from vertex $x$ to vertex $y$ that does not contain edge $(u, v)$ ?" Here, we are concerned with general weighted digraphs and distance queries instead of reachability queries, and we consider both vertex and edge failures.

This problem is similar to the replacement paths problem [11] (see also the erratum for [11] and $[12,18,21]$ ) which, given a pair of vertices $x$ and $y$ in $G$, computes the set of shortest paths from $x$ to $y$ avoiding each of the vertices (or edges) on $\pi_{x y}$ one at a time, where $\pi_{x y}$ is the original shortest path from $x$ to $y$ in $G$. A method for solving this problem on undirected graphs in $O(m+n \log n)$ preprocessing time and $O(n)$ space is given for the vertex removal case in [18] and for the edge removal case in [11]. A method for solving this problem on unweighted directed graphs in $\tilde{O}(m \sqrt{n})$ preprocessing time and $\tilde{O}\left(n^{3 / 2}\right)$ space is given in [21].

However, in this paper we are interested in finding replacement paths for all possible sources and destinations, blowing up each of the above bounds by a factor $n^{2}$. In particular, the space becomes $O\left(n^{3}\right)$. Moreover, we consider the general case of a weighted directed graph. 
Of a similar flavor is the most vital node (or arc) problem $[1,2,4,18]$, which is the problem of identifying the vertex (or edge) on a given shortest path, whose removal results in the longest replacement path.

The most natural approach to the distance sensitivity problem would be to use one of the recent dynamic all pairs shortest paths (APSP) algorithms $[5,6,13,15]$ and delete the failed vertex or edge. The best bounds [6, 25] take $\widetilde{O}\left(n^{2}\right)$ amortized time for real weighted directed graphs, but then queries avoiding the failed vertex or edge are answered in constant time. However, our goal here is to answer a query as quickly as possible after a vertex or edge failure, and then it may be faster to compute the answer from scratch at each query using an $O(n \log n+m)$ single-source shortest paths (SSSP) algorithm [10].

Another extreme solution would be to construct a table that for each vertex pair $(x, y)$ and each vertex/edge stores the distance from $x$ to $y$ avoiding that vertex/edge. For vertex failures, such a table of size $O\left(n^{3}\right)$ is trivially computed by $n$ APSP computations in $O\left(m n^{2}+n^{3} \log n\right)$ time. However, for edge failures the size of the trivial table is $\Theta\left(m n^{2}\right)$ and requires $m$ APSP computations. Space can be reduced (at least for the edge failure case) by working from one source $x$ at a time, constructing a shortest paths tree $T(x)$. This tree changes only if we remove any of the $O(n)$ vertices or edges in it. Hence, it is only for these vertices and edges that we need to record new distances from $x$. An implementation of this idea is given in [24]. If $h$ is the maximal hop count of a path in $T(x)$, the construction uses $h$ SSSP computations in $O(h m+h n \log n)$ time and $O(h n)$ space. However, we may have $h=\Omega(n)$, and repeating the construction from all sources, we end up with a construction time of $O\left(m n^{2}+n^{3} \log n\right)$ and a space bound of $O\left(n^{3}\right)$ for our all pairs case. The fundamental question considered here is if the cubic space bound can be improved.

Shortest paths computation. In the above time bounds, we have assumed that SSSP is solved in $O(m+n \log n)$ time using Fibonacci heaps in Dijkstra's SSSP algorithm [10] and that APSP is solved with an SSSP from each vertex in $O(m n+$ $\left.n^{2} \log n\right)$ time. We will make the same assumptions when stating our own results.

We note here that there are alternative faster algorithms in different situations. For example, if the weights are represented as integer or floating point numbers, we can compute SSSP in $O(m+n \log \log n)$ time [26], or even in $O(m)$ time if the graph is undirected [23]. In the case of real weights, we can solve APSP in $O\left(m n+n^{2} \log \log n\right)$ time for sparse graphs [20] and in $O\left(n^{3} \sqrt{\log \log n} / \log n\right)$ time for dense graphs [22, 27].

However, the above improvements are "only" by logarithmic factors, and in our results for distance sensitivity oracles, we are aiming at polynomial improvements in space and construction time. Hence we are satisfied just stating the time bounds assuming Fibonacci heaps in Dijkstra's algorithm.

1.3. Our results. The main result of this article is a deterministic oracle with fast query time for both vertex and edge failures that uses nearly the same space as that required for storing the distance matrix of the input graph. More precisely, we construct an oracle that uses $O\left(n^{2} \log n\right)$ space and answers distance queries subject to a vertex or edge failure in $O(1)$ worst-case time. This result is quite surprising, since the space bound is significantly smaller than $\Theta\left(n^{3}\right)$ and yet our scheme answers queries in $O(1)$ time. We also present an $\Omega(m)$ space lower bound for the single-source version of the problem. Since $m$ can be as high as $\Omega\left(n^{2}\right)$, our oracle is thus almost space-optimal.

The construction time for our oracle is $O\left(m n^{2}+n^{3} \log n\right)$ in the worst case. However, if one is willing to settle for $\Theta\left(n^{2.5}\right)$ space, we can improve the preprocessing 
TABLE 1.1

Known results and our contribution.

\begin{tabular}{|c|c|c|c|c|c|}
\hline References & Context & $\begin{array}{c}\text { Graph } \\
\text { type }\end{array}$ & $\begin{array}{c}\text { Construction } \\
\text { time }\end{array}$ & Space & $\begin{array}{c}\text { Query } \\
\text { time }\end{array}$ \\
\hline Nardelli et al. [18] & $\begin{array}{l}\text { Most vital node } \\
\text { detection } \\
{[\mathrm{v} \text {-dist }(x, y, v)]}\end{array}$ & Undirected & $O\left(m n^{2}+n^{3} \log n\right)$ & $O\left(n^{3}\right)$ & $O(1)$ \\
\hline $\begin{array}{l}\text { Hershberger } \\
\& \text { Suri [11] }\end{array}$ & $\begin{array}{l}\text { Vickrey pricing } \\
\text { in networks } \\
{[\mathrm{e}-\mathrm{dist}(x, y, u, v)]}\end{array}$ & Undirected & $O\left(m n^{2}+n^{3} \log n\right)$ & $O\left(n^{3}\right)$ & $O(1)$ \\
\hline \multirow{4}{*}{ Our contribution } & \multirow{4}{*}{$\begin{array}{c}\text { Vertex/edge failure } \\
{[\mathrm{v}-\operatorname{dist}(x, y, v)]} \\
{[\mathrm{e}-\operatorname{dist}(x, y, u, v)]}\end{array}$} & \multirow{4}{*}{$\begin{array}{c}\text { Directed } \\
\text { (or undirected) }\end{array}$} & \multicolumn{3}{|c|}{ Method 1} \\
\hline & & & $O\left(m n^{2}+n^{3} \log n\right)$ & $O\left(n^{2} \log n\right)$ & $O(1)$ \\
\hline & & & \multicolumn{3}{|c|}{ Method 2} \\
\hline & & & $O\left(m n^{1.5}+n^{2.5} \log n\right)$ & $O\left(n^{2.5}\right)$ & $O(1)$ \\
\hline
\end{tabular}

time to $O\left(m n^{1.5}+n^{2.5} \log n\right)$, and the query time remains constant. In Table 1.1 we place our bounds in perspective by comparing them to the bounds for related problems obtainable with previous algorithms: in the context of most vital node detection and Vickrey pricing, we are extrapolating the performance of algorithms designed for a single source-destination pair to the all pairs case.

To achieve our bounds, we construct data structures where we store information about APSP excluding only vertices or edges with specific properties, rather than excluding all possible vertices or edges. We also store information about shortest paths where we exclude vertices on entire subpaths, rather than single vertices. We remark that our algorithms are very simple and thus amenable to efficient implementations.

We note that vertex failures in directed graphs trivially subsume vertex failures in undirected graphs, but the same does not hold for link failures as an undirected link failure corresponds to two symmetric directed link failures. However, our solutions happen to work for the undirected case as well.

Part of the results presented in this paper for link failures were presented in two conference papers: one by the first two authors [7] which presented the two algorithms, and the other by the last two authors [3]. The paper [3] improves the query time from [7], but it also has an extra claim on construction time which is incorrect. Additionally, this paper presents results obtained by the last two authors on extending the oracles in $[7,3]$ to efficiently handle node failures in addition to link failures. This paper also presents a lower bound result obtained by the first two authors on the space requirement for the single-source version of the distance oracle problem.

1.4. Vickrey pricing in networks. The Vickrey mechanism is a generalization of the sealed bid second price auction, in which the highest bidder wins the auction but pays a price equal to the second highest bid. This auction protocol motivates a rational bidder to bid truthfully [17]. In a distributed network in which multiple rational selfinterested agents own different parts of the network, Vickrey mechanism is often the best way to determine the utility of various network elements. In order to elicit truthful responses from the agents, each agent is compensated in proportion to the marginal utility he/she brings to the network. Willing manipulations by participating 
TABLE 1.2

Complexity of computing Vickrey payments for all vertex pairs.

\begin{tabular}{|c|c|c|c|}
\hline Algorithm & Time & Space & Query time \\
\hline Naïve & $O\left(n^{2}(m+n \log n)\right)$ & $O\left(n^{3}\right)$ & $O(1)$ \\
\hline Our 1st result & $O\left(n^{2}(m+n \log n)\right)$ & $O\left(n^{2} \log n\right)$ & $O(1)$ \\
\hline Our 2nd result & $O\left(n^{1.5}(m+n \log n)\right)$ & $O\left(n^{2.5}\right)$ & $O(1)$ \\
\hline
\end{tabular}

agents is eliminated by making an agent's payment depend only on the declarations of other agents.

Consider a scenario in which we need to find shortest paths in a network $G$, where links are owned by self-interested agents. Agents are assumed to bid on each link individually. Nisan and Ronen [19] formulated the following expression as the payment $p^{e}(x, y)$ to be made to the owner of a link $e$ for a given vertex pair $(x, y)$ :

$$
p^{e}(x, y)= \begin{cases}d_{x y}\left(\left.G\right|_{w_{e}=\infty}\right)-d_{x y}\left(\left.G\right|_{w_{e}=0}\right) & \text { if } e \in \pi_{x y} \\ 0 & \text { otherwise }\end{cases}
$$

where $\pi_{x y}$ is a shortest path from vertex $x$ to vertex $y$ in $G$, and $d_{x y}\left(\left.G\right|_{w_{e}=k}\right)$ is the distance from vertex $x$ to vertex $y$ in $G$, where the weight of edge $e$ is set to $k$. For any $e \in \pi_{x y}$, the term $d_{x y}\left(\left.G\right|_{w_{e}=0}\right)$ can be simply computed as $d_{x y}\left(\left.G\right|_{w_{e}=0}\right)=d_{x y}-w_{e}$, where $d_{x y}$ is the distance from $x$ to $y$ in $G$. However, computing $d_{x y}\left(\left.G\right|_{w_{e}=\infty}\right)$ naïvely for all $e \in \pi_{x y}$ requires running an $O(m+n \log n)$ time shortest paths algorithm [8, 10] on $G-\{e\}$ for each $e \in \pi_{x y}$. This can be as high as $O\left(m n+n^{2} \log n\right)$ in the worst case. This problem was studied in [11], but no improvement to this trivial bound is known.

The distance sensitivity problem we study in this article is a generalization of the above problem to the situation where one is potentially interested in finding all Vickrey payments for all vertex pairs (instead of a single pair). In this case our first algorithm can carry out the entire computation using significantly less space than that used by the naïve algorithm. On the other hand, our second algorithm reduces both time and space requirements of the computation. The complexities of all three algorithms are compared in Table 1.2. Observe, however, that for very sparse graphs the running time of the naïve algorithm can be improved to $O(m n(m+n \log \log n))$ [20].

1.5. Organization of the article. The remainder of this article is organized as follows. In section 2 we introduce the notation used in the article, and we discuss some simple properties that will be useful in the description of our results. In particular, we define the notion of "path cover," showing how to use information about shortest paths which avoid all vertices on certain paths in the graph to determine a shortest path that avoids a single vertex. In section 3 we show how to efficiently compute shortest paths from any given vertex to all other vertices in a directed graph $G$ with nonnegative real-valued edge weights where we avoid all vertices on certain 
types of paths. These tools are used in section 4 to devise an oracle for the distance sensitivity problem that answers $\mathrm{v}$-dist queries in constant worst-case time using nearly the same space required for storing a single distance matrix. This oracle can be constructed in $O\left(m n^{2}+n^{3} \log n\right)$ worst-case time. In section 5 we show that, if one is willing to settle for more space, we can reduce the preprocessing time to $O\left(m n^{1.5}+n^{2.5} \log n\right)$. This second oracle uses $O\left(n^{2.5}\right)$ space while still answering $\mathrm{v}$-dist queries in constant worst-case time. Section 6 shows how to extend the oracles designed for vertex failures to also deal with edge failures, and section 7 addresses the problem of supporting path queries v-path and e-path. A space lower bound for the single-source version of the distance sensitivity problem is discussed in section 8 . Finally, section 9 provides some concluding remarks.

2. Preliminaries. Let $G=(V, E, w)$ be a directed graph with vertex set $V$, edge set $E$, and edge weight function $w$. Throughout the article, we assume that, for each pair of vertices $x$ and $y$ such that $y$ is reachable from $x$, there is a unique shortest path from $x$ to $y$. This is without loss of generality, since ties can be broken arbitrarily (see, e.g., [6]).

2.1. Notation. In this article, a path $p_{x y}$ is a sequence of vertices of the form $p_{x y}=\left\langle v_{0}, v_{1}, v_{2}, \ldots, v_{k-1}, v_{k}\right\rangle$ such that $v_{0}=x, v_{k}=y$, and $\left(v_{i}, v_{i+1}\right) \in E$ for every $i, 0 \leq i<k$. Thus, $G-p_{x y}$ is the subgraph of $G$ induced by the vertex set $V-\left\{x, v_{1}, \ldots, v_{k-1}, y\right\}$. We let $p_{x y} \cdot p_{y z}$ denote the concatenation of path $p_{x y}$ with path $p_{y z}$. We denote by $w_{x y}$ the weight of edge $(x, y)$ in $G$, and we indicate with $w\left(p_{x y}\right)$ the length of $p_{x y}$, i.e., the sum of weights of edges in $p_{x y}$. We also denote by $T(x)$ the single-source shortest path tree of $G$ with source $x$, and we denote by $\pi_{x y}$ the (unique) shortest path from vertex $x$ to vertex $y$ in $G$, if any. Using a geometrical analogy, we sometimes look at shortest paths as "segments," using the notation $[x, y]$ instead of $\pi_{x y}$. Similarly, we sometimes use $] x, y\left[\right.$ to denote $\pi_{x y}-\{x, y\},[x, y[$ to denote $\pi_{x y}-\{y\}$, and $\left.] x, y\right]$ to denote $\pi_{x y}-\{x\}$. We indicate with $d_{x y}$ the length of $\pi_{x y}$ (distance from $x$ to $y$ in $G$ ), and with $h_{x y}$ the number of edges in $\pi_{x y}$ (number of hops). If $y$ is not reachable from $x$, we assume that $d_{x y}=h_{x y}=+\infty$. By $\widehat{G}$ we denote the directed graph obtained from $G$ by reversing the orientation of edges. Thus, $\widehat{\pi}$ and $\widehat{T}$ denote $\pi$ and $T$ in $\widehat{G}$. If $T$ is a rooted tree, we let $B_{T}(i, j)$ denote the set of paths in $T$ that connect vertices at level $i$ with vertices at level $j>i$ in the tree, assuming that the root of $T$ has level 0 . Notice that, if $\pi_{c d} \in B_{T(x)}(i, j)$, then $h_{c d}=j-i$. Let $a$ and $b$ be vertices on $p_{x y}$; we say that $a<b$ if $a$ appears before $b$ on $p_{x y}$, and $a \leq b$ if $a<b$ or $a=b$. Finally, by $\pi_{x y}^{u v}$ we denote a shortest path from vertex $x$ to vertex $y$ in $G-[u, v]$. The path $\pi_{x y}^{u v}$ could be thought of as an optimal "replacement path" from $x$ to $y$ to be used in case all vertices in $[u, v]$ fail. Observe that, for each internal vertex $v$ in $\pi_{x y}, \mathrm{v}$-dist $(x, y, v)=w\left(\pi_{x y}^{v v}\right)$. Indeed, the goal of this article is to provide methods for answering queries about $\pi_{x y}^{v v}$. The notation used in this article is summarized in Table 2.1.

2.2. Structural properties. By our assumption of uniqueness of shortest paths, for any pair of vertices $a, b \in \pi_{x y}$ such that $a \leq b, \pi_{a b}$ is a subpath of $\pi_{x y}$. Moreover, if $\pi_{x y}=\left\{v_{0}, v_{1}, \ldots, v_{k-1}, v_{k}\right\}$, then $\hat{\pi}_{y x}=\left\{v_{k}, v_{k-1}, \ldots, v_{1}, v_{0}\right\}$; i.e., one is the reversal of the other.

We now discuss a simple structural property of $\pi_{x y}^{u v}$. In particular, the following claim shows that, if $y$ is reachable from $x$ in $G-[u, v]$, then $\pi_{x y}^{u v}$ and $\pi_{x y}$ share a common prefix and a common suffix and are vertex-disjoint elsewhere (see Figure 2.1). Intuitively, the internal subpath of $\pi_{x y}^{u v}$ that is vertex-disjoint from $\pi_{x y}$ can be thought 
TABLE 2.1

Notation used in this article.

\begin{tabular}{|c|l|}
\hline Notation & \\
\hline$G$ & edge-weighted directed graph $G=(V, E, w)$ \\
\hline$w_{x y}$ & weight of edge $(x, y)$ in $G$ \\
\hline$p_{x y}$ & path $\left\langle x, v_{1}, v_{2}, \cdots, v_{k-1}, y\right\rangle$ from vertex $x$ to vertex $y$ in $G$ \\
\hline$p_{x y} \cdot p_{y z}$ & concatenation of path $p_{x y}$ with path $p_{y z}$ \\
\hline$w\left(p_{x y}\right)$ & length of path $p_{x y}\left(\right.$ sum of weights of edges in $\left.p_{x y}\right)$ \\
\hline$\pi_{x y}$ or $[x, y]$ & shortest path from vertex $x$ to vertex $y$ in $G$ (we assume it is unique) $)$ \\
\hline$] x, y[$ & $\pi_{x y}-\{x, y\}$ \\
\hline$[x, y[$ & $\pi_{x y}-\{y\}$ \\
\hline$] x, y]$ & $\pi_{x y}-\{x\}$ \\
\hline$h_{x y}$ & number of edges in $\pi_{x y}$ \\
\hline$d_{x y}$ & distance from vertex $x$ to vertex $y$ in $G \quad\left(d_{x y}=w\left(\pi_{x y}\right)\right)$ \\
\hline$T(x)$ & shortest path tree rooted at $x$ in $G(x$ is at level 0 in $T(x))$ \\
\hline$\widehat{G}$ & reversed graph $(V, \widehat{E}, \widehat{w})$ s.t. $\widehat{E}=\{(x, y):(y, x) \in E\}$ and $\widehat{w}_{x y}=w_{y x}$ \\
\hline$\widehat{T}(x), \widehat{\pi}_{x y}$ & $T(x)$ and $\pi_{x y}$ in $\widehat{G}$ instead of $G$ \\
\hline$B_{T}(i, j)$ & set of all paths in tree $T$ connecting vertices at level $i$ to vertices at level $j$ \\
\hline$a<b$ in $p_{x y}$ & vertex $a$ precedes vertex $b$ in $p_{x y}$ \\
\hline$\pi_{x y}^{u v}$ & shortest path from $x$ to $y$ in $G-[u, v]$ \\
\hline$d_{x y}\left(l_{1}, l_{2}, r_{1}, r_{2}\right)$ & min $a \in\left[l_{1}, l_{2}\left[\left\{d_{x a}+w\left(\pi_{a b}^{l_{2} r_{1}}\right)+d_{b y}\right\}\right.\right.$ \\
\hline \\
\hline
\end{tabular}

of as an "optimal detour" that avoids vertices in $[u, v]$.

Claim 1. Let $G=(V, E, w)$ be an edge-weighted directed graph. For any $x, y \in V$ and for any $u, v \in \pi_{x y}$ with $x<u \leq v<y$, if $\pi_{x y}^{u v} \neq \emptyset$, then there exist two vertices $a, b \in \pi_{x y}$ such that $\pi_{x y}^{u v}=\pi_{x a} \cdot p_{a b} \cdot \pi_{b y}$, where $p_{a b} \cap \pi_{a b}=\{a, b\}$.

Proof. We first notice that $\pi_{x y}$ and $\pi_{x y}^{u v}$ are both paths in $G$, since every path in $G-[u, v]$ is also a path in $G$. Moreover, $\pi_{x y} \neq \pi_{x y}^{u v}$, since $u \in \pi_{x y}$, but $u \notin \pi_{x y}^{u v}$.

Now, let $p_{x a}$ be the longest common prefix of $\pi_{x y}^{u v}$ and $\pi_{x y}$, and let $p_{b y}$ be their longest common suffix. These subpaths are never empty, since $\pi_{x y}^{u v}$ and $\pi_{x y}$ share at least their endpoints. This proves the existence of vertices $a$ and $b$ in our claim.

Since every subpath of $\pi_{x y}$ is a shortest path (by the optimal substructure property of shortest paths) and shortest paths are unique in $G$, then $p_{x a}=\pi_{x a}$ and $p_{b y}=\pi_{b y}$. Furthermore, since $\pi_{x y} \neq \pi_{x y}^{u v}$, then $a \neq b$ and $p_{x a} \cap p_{b y}=\emptyset$. Thus, we can write $\pi_{x y}^{u v}$ as $\pi_{x a} \cdot p_{a b} \cdot \pi_{b y}$ for some $p_{a b}$.

It remains to prove that $p_{a b} \cap \pi_{a b}=\{a, b\}$. Suppose by contradiction that there is a vertex $c \in p_{a b} \cap \pi_{a b}$ such that $a<c<b$. Now, observe that $c \notin[u, v]$, since $p_{a b} \cap[u, v]=\emptyset$, and that $[u, v]$ is a subpath of $\pi_{a b}$, since $\pi_{x a} \cap[u, v]=\emptyset$ and $\pi_{b y} \cap[u, v]=$ $\emptyset$. Assume without loss of generality that $c<u$ in $\pi_{x y}$ (the case $v<c$ is completely analogous). Consider the subpath $\pi_{a c}$ of $\pi_{a b}$, and notice that it is a shortest path in both $G$ and $G-[u, v]$. Now, $p_{a b}$ is a shortest path in $G-[u, v]$, and thus, by the optimal-substructure property, its subpath $p_{a c}$ has to be shortest as well in $G-[u, v]$. Since shortest paths are unique in $G-[u, v]$, then $p_{a c}=\pi_{a c}$, and thus $\pi_{x y}$ and $\pi_{x y}^{u v}$ 


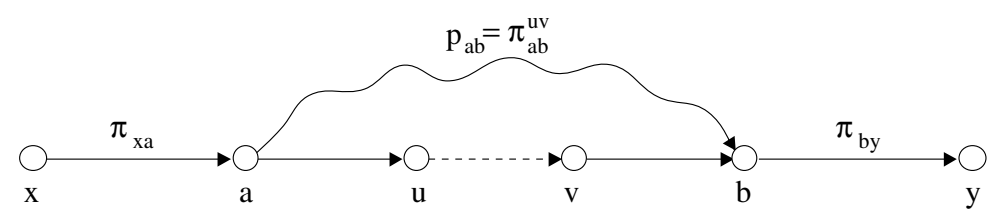

FIG. 2.1. Structure of a replacement path $\pi_{x y}^{u v}=\pi_{x a} \cdot p_{a b} \cdot \pi_{b y}$.

share the same subpath from $x$ to $c>a$. This means that $a$ cannot be the last vertex of the longest common prefix of $\pi_{x y}^{u v}$ and $\pi_{x y}$, which is clearly a contradiction.

2.3. Path covering. We now discuss the notion of "path covering" that will be crucial to proving the correctness of our query algorithms.

Definition 2.1. Let $x \leq l_{1}<l_{2} \leq r_{1}<r_{2} \leq y$ be vertices on $\pi_{x y}$. We define $d_{x y}\left(l_{1}, l_{2}, r_{1}, r_{2}\right)$ as

$$
d_{x y}\left(l_{1}, l_{2}, r_{1}, r_{2}\right)=\min _{\substack{a \in\left[l_{1}, l_{2}[ \\b \in] r_{1}, r_{2}\right]}}\left\{d_{x a}+w\left(\pi_{a b}^{l_{2} r_{1}}\right)+d_{b y}\right\},
$$

and we say that a value $d$ covers $\left[l_{1}, l_{2}[\times] r_{1}, r_{2}\right]$ w.r.t. $x, y$ if $d \leq d_{x y}\left(l_{1}, l_{2}, r_{1}, r_{2}\right)$. In this case, we also say that $d$ covers all paths of the form $\pi_{x a} \cdot \pi_{a b}^{l_{2} r_{1}} \cdot \pi_{b y}$ for each $a \in\left[l_{1}, l_{2}[\right.$ and for each $\left.b \in] r_{1}, r_{2}\right]$.

Observe that $d_{x y}\left(l_{1}, l_{2}, r_{1}, r_{2}\right)$ is the distance from vertex $x$ to vertex $y$ in $G$ using paths that first follow a shortest path from $x$ to some vertex $a$ in $\left[l_{1}, l_{2}[\right.$, then take an optimal detour that avoids all vertices in $\left[l_{2}, r_{1}\right]$, and finally go through a shortest path from some vertex $b$ in $\left.] r_{1}, r_{2}\right]$ to $y$. The following claim, which easily follows from Definition 2.1 and from Claim 1, states that, if $l_{1}=x$ and $r_{2}=y$, then $d_{x y}\left(l_{1}, l_{2}, r_{1}, r_{2}\right)$ is equal to the length of the shortest path from $x$ to $y$ avoiding $[u, v]$.

Claim 2. Let $x<u \leq v<y$ be vertices on $\pi_{x y}$. Then $w\left(\pi_{x y}^{u v}\right)=d_{x y}(x, u, v, y)$.

We now show how information about the distance from $x$ to $y$ avoiding $[u, v]$ with detours having constrained positions of their endpoints can be used to compute $w\left(\pi_{x y}^{u v}\right)$. The following claim will be useful to prove the correctness of our query algorithms.

Claim 3. For any $x<\widehat{u} \leq \widetilde{u}<u \leq v<\widetilde{v} \leq \widehat{v}<y$ on $\pi_{x y}$,

$$
d_{x y}(x, u, v, y)=\min \left\{d_{x y}(x, \widetilde{u}, \widetilde{v}, y), d_{x y}(\widehat{u}, u, v, y), d_{x y}(x, u, v, \widehat{v})\right\} .
$$

Proof. Let the endpoints of the optimal detour in $\pi_{x y}^{u v}$ be $a$ and $b, a<b$, and consider all possible positions of $a$ in $[x, u[$ :

- $a$ in $\left[x, \widehat{u}\left[: d_{1}=d_{x y}(x, u, v, \widehat{v})\right.\right.$ handles the cases when $b$ lies in $\left.] v, \widehat{v}\right]$; i.e., $d_{1}$ covers $[x, \widehat{u}[\times] v, \widehat{v}]$ w.r.t. $x, y$. Moreover, $d_{2}=d_{x y}(x, \widetilde{u}, \widetilde{v}, y)$ handles the cases when $b$ lies in $] \widehat{v}, y]$; i.e., $d_{2}$ covers $[x, \widehat{u}[\times] \widehat{v}, y]$ w.r.t. $x, y$. Thus, $d_{3}=\min \left\{d_{x y}(x, u, v, \widehat{v}), d_{x y}(x, \widetilde{u}, \widetilde{v}, y)\right\}$ handles all possible positions of $b$ in ]$v, y]$; i.e., $d_{3}$ covers $[x, \widehat{u}[\times] v, y]$ w.r.t. $x, y$.

- $a$ in $\left[\widehat{u}, u\left[: d_{4}=d_{x y}(\widehat{u}, u, v, y)\right.\right.$ handles the cases where $b$ lies in $\left.] v, y\right]$; i.e., $d_{4}$ covers $[\widehat{u}, u[\times] v, y]$ w.r.t. $x, y$.

Thus, for each possible position of $a$ in $\left[x, u\left[, d_{5}=\min \left(d_{x y}(x, \widetilde{u}, \widetilde{v}, y), d_{x y}(\widehat{u}, u, v, y)\right.\right.\right.$, $\left.d_{x y}(x, u, v, \widehat{v})\right)$ handles all possible positions of $b$ in $\left.] v, y\right]$; i.e., $d_{5}$ covers $[x, u[\times] v, y]$ w.r.t. $x, y$. Since $d_{5}$ equals the length of some path from $x$ to $y$ in $G-[u, v]$, we can then conclude that $d_{5}=d_{x y}(x, u, v, y)$. 
3. Distances under deletion of paths. In this section we provide simple algorithms for computing distances in a directed graph $G$ with nonnegative realvalued edge weights where we avoid all vertices on certain paths. These algorithms will be useful in sections 4 and 5 for constructing distance sensitivity oracles.

Let $x$ be a vertex and let $P$ be a set of shortest paths in $G$. We consider the problem of designing a procedure exclude $(G, x, P)$ that computes for each path $\pi \in P$ the distances from vertex $x$ to all other vertices in $G-\pi$. Throughout this article we assume that the deletion of the vertices on a given path $\pi$ results in the deletion of all its vertices including its endpoints.

We can compute exclude $(G, x, P)$ with a straightforward algorithm that runs in $O(|P|(m+n \log n))$ worst-case time using a Dijkstra computation [10] on the graph $G$ with all vertices in $\pi$ deleted for each $\pi \in P$. In the remainder of this section we show that this computation can be made considerably more efficient if we restrict our attention to $P \subseteq T(x)$ (i.e., every path in $P$ is also a path in $T(x))$ and if we assume that paths in $P$ are "independent," a notion we define in Definition 3.1.

3.1. Algorithm fast-exclude. In this section we devise a variant of exclude $(G, x, P)$, which we call fast-exclude $(G, x, P)$, for the case when $P \subseteq T(x)$. As above, our goal is to compute for each path $\pi \in P$ the distances from vertex $x$ to all other vertices in $G-\pi$.

Let $P \subseteq T(x)$, and for any path $\pi \in P$, denote by $T_{x}(\pi)$ the subtree of $T(x)$ rooted at the first vertex of $\pi$, and let $W_{\pi}$ be the set of all vertices in $T_{x}(\pi)$ except the vertices on $\pi$. We observe that only vertices in $W_{\pi}$ may have their distances from $x$ increased if we remove from $G$ the vertices on $\pi$. Now, consider the following directed graph $G_{\pi}=\left(V_{\pi}, E_{\pi}, w_{\pi}\right)$, where the following hold:

- $V_{\pi}=W_{\pi} \cup\{x\}$.

- $E_{\pi}$ contains an edge from $x$ to each vertex in $W_{\pi}$ and the edges in $G$ induced by vertices in $W_{\pi}$.

- $w_{\pi, a b}$ is the weight of edge $(a, b)$ in $G_{\pi}$ defined as

$$
w_{\pi, a b}= \begin{cases}\min _{c \notin T_{x}(\pi)}\left\{d_{x c}+w_{c b}\right\} & \text { if } a=x, \\ w_{a b} & \text { otherwise, }\end{cases}
$$

where we assume that $d_{x c}=+\infty$ if $c$ is not reachable from $x$ in $G$ and $w_{c b}=+\infty$ if $(c, b)$ is not an edge of $G$.

It is not difficult to see (see proof of Claim 4) that the shortest path from vertex $x$ to a vertex $v$ in $W_{\pi}$ has the same length in $G-\pi$ as the shortest path from $x$ to $v$ in $G_{\pi}$. Hence, distances in $G-\pi$ from $x$ to all vertices in $W_{\pi}$ can be computed by a Dijkstra computation on $G_{\pi}$ with source $x$. The algorithm fast-exclude $(G, x, P)$ works in the same way as exclude $(G, x, P)$, but it uses $G_{\pi}$ instead of $G$ for each $\pi$ in $P$.

Since the graph $G_{\pi}$ is typically smaller than $G$, fast-exclude can be expected to have better performance than exclude for any collection of paths $P \subseteq T(x)$. We now define the notion of independent shortest paths, and we show that fast-exclude performs significantly better than exclude when $P \subseteq T(x)$ is a collection of independent shortest paths.

DeFinition 3.1. Let $T$ be a rooted tree and let $\pi_{u v}$ and $\pi_{u^{\prime} v^{\prime}}$ be two paths in $T$. We say that $\pi_{u v}$ and $\pi_{u^{\prime} v^{\prime}}$ are independent in $T$ if the subtree of $T$ rooted at $u$ and the subtree of $T$ rooted at $u^{\prime}$ are disjoint.

ClaIM 4. If $P \subseteq T(x)$ is a set of pairwise independent shortest paths in $T(x)$, then algorithm fast-exclude $(G, x, P)$ requires $O(m+n \log n)$ time in the worst case 
and computes the same output as exclude $(G, x, P)$.

Proof. Using the notation given above, for a given $\pi \in P$, let $\pi_{x y}^{\prime}$ be the shortest path from $x$ to any $y \in W_{\pi}$ avoiding the vertices on $\pi$. Let $b$ be the first vertex on $\pi_{x y}^{\prime}$ such that $b \in W_{\pi}$, and let $c$ be the vertex preceding $b$. Then let us write $\pi_{x y}^{\prime}=p_{x c} \cdot\langle c, b\rangle \cdot \pi^{\prime}{ }_{b y}$, where $\langle c, b\rangle$ is the path from $c$ to $b$ formed by the single edge $(c, b)$. Since for any $z \notin T_{x}(\pi)$, the path $\pi_{x z}$ is composed entirely of the vertices in $T(x)-T_{x}(\pi)$, it follows that $p_{x c}=\pi_{x c}$. Also note that $\pi_{b y}^{\prime}$ cannot contain any vertex $z \notin T_{x}(\pi)$, since if it contains such a vertex $z$, then $\pi_{x z}$ will be a shorter path to $z$, contradicting our choice of $b$. These two observations justify the use of $G_{\pi}$ instead of $G-\pi$ in order to compute the shortest path tree rooted at $x$ avoiding the vertices on $\pi$.

For each $\pi \in P,\left|V_{\pi}\right|$ is never greater than the number of vertices in $T_{x}(\pi)$. Since the paths in $P$ are pairwise independent, any two such subtrees are disjoint for distinct paths in $P$. Since each $E_{\pi}$ contains edges in $G$ induced by vertices in $W_{\pi}$ and edges from $x$ to only the vertices in $W_{\pi}$, it follows that the sum of the cardinalities of all $V_{\pi}$ and $E_{\pi}$ are linear in $n$ and $m$, respectively. Hence, fast-exclude $(G, x, P)$ runs in $O(m+n \log n)$ time when $P \subseteq T(x)$ is a set of independent shortest paths.

4. Oracle with $O(1)$ query time and $O\left(n^{2} \log n\right)$ space. In this section we describe a deterministic oracle for single-vertex failure with constant query time that uses nearly the same space as that required for storing a single distance matrix. In particular, we show how to preprocess a graph with nonnegative real-valued edge weights in $O\left(m n^{2}+n^{3} \log n\right)$ worst-case time, producing a compact oracle that uses $O\left(n^{2} \log n\right)$ space and answers v-dist queries in $O(1)$ worst-case time.

4.1. Data structure. Using $O\left(n^{2} \log n\right)$ space, we maintain each $d_{x y}$ and $h_{x y}$, and we maintain six matrices $d l, d r, s l, s r, v l$, and $v r$ of size $n \times n \times\left\lfloor\log _{2} n\right\rfloor$ defined for every pair of distinct vertices $x$ and $y$ as follows:

- $d l[x, y, i]=$ distance from vertex $x$ to vertex $y$ in $G-\pi$, where $\pi$ is the subpath of $\pi_{x y}$ starting at level $2^{i-1}$ and ending at level $2^{i}-1$ in $T(x)$, and $1 \leq i \leq \log _{2} h_{x y}$

- $d r[x, y, i]=$ distance from vertex $y$ to vertex $x$ in $\widehat{G}-\pi$, where $\pi$ is the subpath of $\widehat{\pi}_{y x}$ starting at level $2^{i-1}$ and ending at level $2^{i}-1$ in $\widehat{T}(y)$, and $1 \leq i \leq \log _{2} h_{x y}$

- $s l[x, y, i]=$ distance from vertex $x$ to vertex $y$ in $G-\{v\}$, where $v$ is the vertex of $\pi_{x y}$ at level $2^{i-1}$ in $T(x)$, and $1 \leq i<1+\log _{2} h_{x y}$;

- $s r[x, y, i]=$ distance from vertex $y$ to vertex $x$ in $\widehat{G}-\{v\}$, where $v$ is the vertex of $\widehat{\pi}_{y x}$ at level $2^{i-1}$ in $\widehat{T}(y)$, and $1 \leq i<1+\log _{2} h_{x y}$;

- $v l[x, y, i]=$ vertex of $\pi_{x y}$ at level $2^{i-1}$ in $T(x)$, where $1 \leq i \leq 1+\log _{2} h_{x y}$;

- $\operatorname{vr}[x, y, i]=$ vertex of $\widehat{\pi}_{y x}$ at level $2^{i-1}$ in $\widehat{T}(y)$, where $1 \leq i \leq 1+\log _{2} h_{x y}$.

4.2. Preprocessing. The above quantities are computed in the preprocessing phase as follows:

- Distances $d_{x y}$ and $h_{x y}$ and matrices $v l$ and $v r$ are easily initialized from shortest path trees of $G$.

- To compute $d l[x, y, i]$, we call procedure exclude $\left(G, x, B_{T(x)}\left(2^{i-1}, 2^{i}-1\right)\right)$, discussed in section 3 , for each $x$ and for each $i, 1 \leq i<\log _{2} n$.

- To compute $d r[x, y, i]$, we call procedure $\operatorname{exclude}\left(\widehat{G}, y, B_{\widehat{T}(y)}\left(2^{i-1}, 2^{i}-1\right)\right)$, for each $y$ and for each $i, 1 \leq i<\log _{2} n$. 


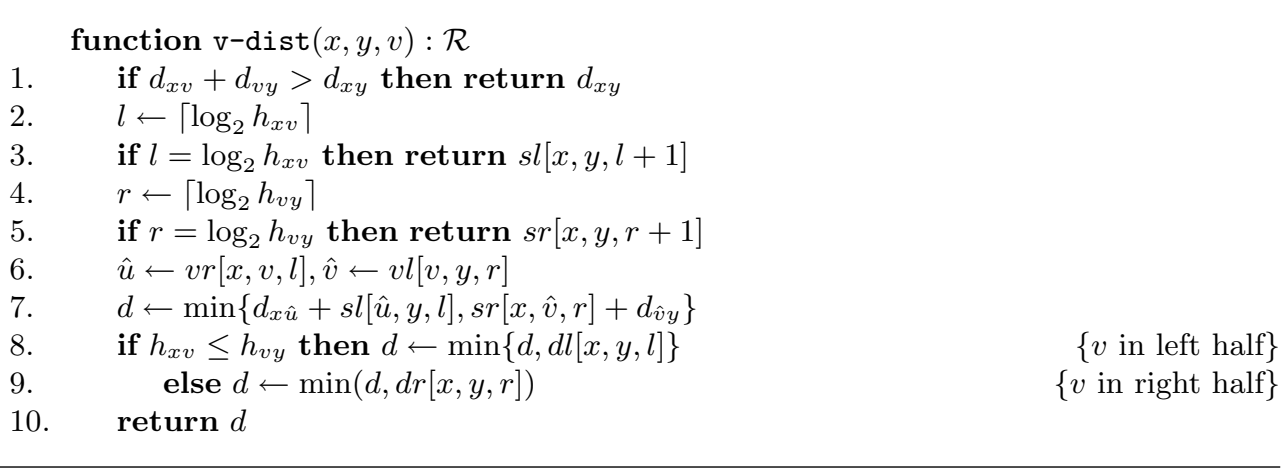

FIG. 4.1. Query algorithm for the first oracle.

- For each $x$ and for each $i, 1 \leq i<1+\log _{2}(n-1)$, we compute $s l[x, y, i]$ by calling procedure fast-exclude $\left(G, x, B_{T(x)}\left(2^{i-1}, 2^{i-1}\right)\right)$.

- We compute $\operatorname{sr}[x, y, i]$ by calling procedure fast-exclude $\left(\widehat{G}, y, B_{\widehat{T}(y)}\left(2^{i-1}\right.\right.$, $\left.2^{i-1}\right)$ ) for each $y$ and for each $i, 1 \leq i<1+\log _{2}(n-1)$.

4.3. Query. The query algorithm is shown in Figure 4.1. We address only the general interesting case where $v \neq x$ and $v \neq y$; otherwise, the answer is clearly $+\infty$. In line 1 of the algorithm, we get rid of the case where $v \notin \pi_{x y}$ and return $d_{x y}$ as the answer. Lines 2 and 3 take care of the case where $v$ is $2^{l}$ edges away from vertex $x$ on $\pi_{x y}$ for some nonnegative integer $l, 0 \leq l<\log _{2} h_{x y}$. Lines 4 and 5 handle the case where $v$ is $2^{r}$ edges away from vertex $y$ on $\widehat{\pi}_{y x}$ for some nonnegative integer $r$, $0 \leq r<\log _{2} h_{x y}$. Lines 6 to 9 take care of the remaining cases.

4.4. Analysis. We first discuss the correctness of our query procedure. Using the matrices $s l$ and $s r$, lines 2 to 5 of the query algorithm answer the following two types of trivial queries: (1) $h_{x v}=2^{l}$ for some nonnegative integer $l, 0 \leq l<\log _{2} h_{x y}$, and (2) $h_{v y}=2^{r}$ for some nonnegative integer $r, 0 \leq r<\log _{2} h_{x y}$. So in order to prove the correctness of $\mathrm{v}$-dist, we need only to prove the correctness of the code segment of lines 6 to 9 that handles the nontrivial case when neither of the above two conditions holds.

In line 7 , we assign $d$ to the minimum of $d_{x \hat{u}}+\operatorname{sl}[\widehat{u}, y, l]$ and $\operatorname{sr}[x, \widehat{v}, r]+d_{\hat{v} y}$, where $d_{x \hat{u}}+s l[\widehat{u}, y, l]=d_{x y}(\widehat{u}, v, v, y)$ and $\operatorname{sr}[x, \widehat{v}, r]+d_{\hat{v} y}=d_{x y}(x, v, v, \widehat{v})$. In line 8 , we consider the case where $h_{x v} \leq h_{v y}$, i.e., $v$ is in the first half of $\pi_{x y}$ (see Figure 4.2). In this case, $0<h_{x \hat{u}}<h_{\hat{u} v}=2^{l-1} \leq h_{v \hat{v}}$. The value $d l[x, y, l]$ is the distance from $x$ to $y$ avoiding a subpath having $2^{l-1}$ vertices and starting at a vertex that is $2^{l-1}$ edges away from $x$ on $\pi_{x y}$. Let the endpoints of that subpath be $\widetilde{u}$ and $\widetilde{v}$, and $h_{x \tilde{u}}<h_{x \tilde{v}}$. So, we have $x<\widehat{u} \leq \widetilde{u}<v<\widetilde{v} \leq \widehat{v}<y$. Thus, by Claim 3, $d$ covers $[x, v[\times] v, y]$ following the assignment in line 8. By construction of matrices $d l, s r$, and $s r, d$ is always equal to the weight of some path from $x$ to $y$ that does not use vertex $v$. Thus, we can conclude that $d$ is the desired answer to $\mathrm{v}$-dist $(x, y, v)$. A similar argument holds for the case where $h_{x v}>h_{v y}$ (line 9).

We now address the running times of preprocessing and query procedures.

Claim 5. Preprocessing requires $O\left(m n^{2}+n^{3} \log n\right)$ worst-case time and any $\mathrm{v}$-dist operation requires $O(1)$ worst-case time.

Proof. We observe that the number of distinct paths in $B_{T(x)}\left(2^{i-1}, 2^{i}-1\right)$ is 

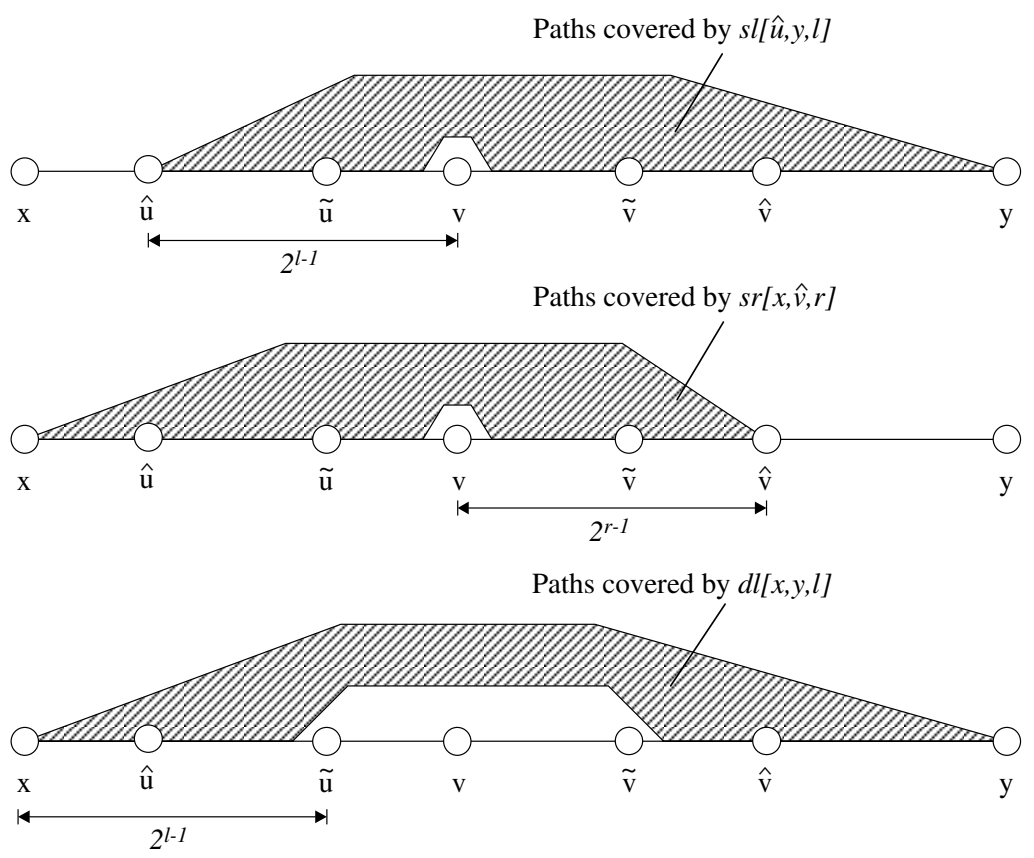

FIG. 4.2. Query example with $h_{x v} \leq h_{v y}$ : The distance from vertex $x$ to vertex $y$ in $G-\{v\}$ can be obtained by taking the minimum of $d_{x \hat{u}}+s l[\widehat{u}, y, l], \operatorname{sr}[x, \widehat{v}, r]+d_{\hat{v} y}$, and $d l[x, y, l]$. Notice that the union of the paths in the grey areas in the figure is the set of all possible detours avoiding vertex $v$.

exactly the same as the number of vertices on level $2^{i}-1$ in $T(x)$. Hence, the total number of distinct paths in all $B_{T(x)}\left(2^{i-1}, 2^{i}-1\right)$ for $1 \leq i<\log _{2} n$ is bounded from above by the number of vertices in $T(x)$. Since exclude $(G, x, P)$ runs in $O(|P|(m+n \log n))$ time and $\sum_{1<i<\log _{2} n}\left|B_{T(x)}\left(2^{i-1}, 2^{i}-1\right)\right|=O(n)$, the matrices $d l$ and $d r$ can be calculated in $O\left(m n^{2}+n^{3} \log n\right)$ worst-case time. On the other hand, for each $x$ and for each $i, 1 \leq i<1+\log _{2}(n-1)$, we can compute $s l[x, y, i]$ by calling procedure fast-exclude $\left(G, x, B_{T(x)}\left(2^{i-1}, 2^{i-1}\right)\right)$ since the singlevertex paths in $B_{T(x)}\left(2^{i-1}, 2^{i-1}\right)$ are trivially pairwise independent in $T(x)$. Since fast-exclude $\left(G, x, B_{T(x)}\left(2^{i-1}, 2^{i-1}\right)\right)$ runs in $O(m+n \log n)$ time, the total time required to compute the matrix $s l$ is $O\left(m n \log n+n^{2} \log ^{2} n\right)$. Similarly the matrix $s r$ can be computed in $O\left(m n \log n+n^{2} \log ^{2} n\right)$ time. Hence the preprocessing time is dominated by the time to compute the $d l$ and $d r$ matrices and requires $O\left(m n^{2}+n^{3} \log n\right)$ worst-case time.

Since the query algorithm executes a constant number of steps, it runs in $O(1)$ worst-case time.

5. Improving the preprocessing time. In this section we show that, if one is willing to settle for more space, we can design a distance sensitivity oracle where we reduce the preprocessing time to $O\left(m n^{1.5}+n^{2.5} \log n\right)$. This second oracle uses $O\left(n^{2.5}\right)$ space and answers distance queries in $O(1)$ worst-case time.

5.1. Data structure. We maintain each $d_{x y}$ and $h_{x y}$, and we maintain five matrices $d h, d t, v c, d c$, and $b c$ using $O\left(n^{2.5}\right)$ space. Matrix $d h$ has size $n \times n \times\lfloor\sqrt{n}\rfloor$, matrices $d t, v c$, and $d c$ have size $n \times n \times\lfloor 2 \sqrt{n}\rfloor$, and matrix $b c$ has size $n \times n$. They 


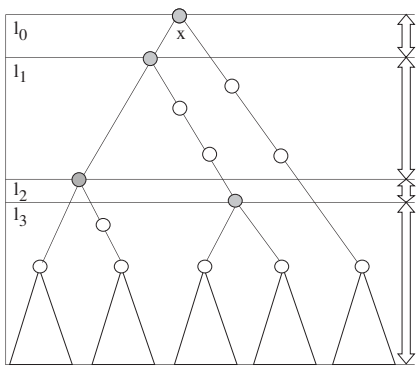

(a) Sequence $\left\{\mathrm{l}_{\mathrm{i}}\right\}$ obtained by cutting $\mathrm{T}^{\prime}(\mathrm{x})$ at vertices of degree $>1$

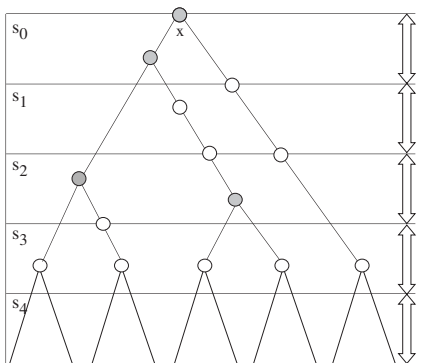

(b) Sequence $\left\{\mathrm{s}_{\mathrm{i}}\right\}$ obtained by cutting at regular intervals of height $\sqrt{n}$

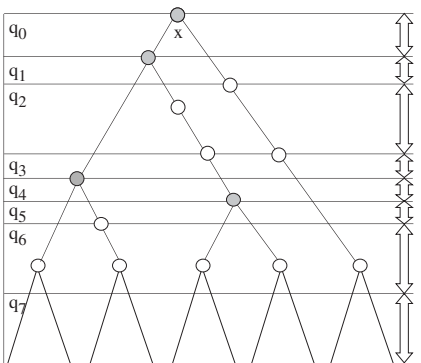

(c) Sequence $\left\{q_{i}\right\}$ obtained by merging sequences $\left\{\mathrm{l}_{\mathrm{i}}\right\}$ and $\left\{\mathrm{s}_{\mathrm{i}}\right\}$

FIG. 5.1. Constructing matrix dc: Cutting tree $T^{\prime}(x)$ to form bands of pairwise independent shortest paths in it.

are defined as follows:

- $d h[x, y, i]=$ distance from vertex $x$ to vertex $y$ in $G-\{v\}$, where $v$ is the vertex of $\pi_{x y}$ at level $i$ in $T(x)$ and $0<i \leq \sqrt{n}$;

- $d t[x, y, i]=$ distance from vertex $x$ to vertex $y$ in $G-\{v\}$, where $v$ is the vertex of $\pi_{x y}$ at level $i$ in $\widehat{T}(y)$ and $0<i \leq 2 \sqrt{n}$;

- $v c[x, y, i]=$ vertex of $\pi_{x y}$ at level $q_{i}$ in $T(x)$, where $q_{0}=0<q_{1}<\cdots<q_{k}<n$ is any increasing sequence of $k+1 \leq 2 \sqrt{n}$ positive numbers depending on $x$, and $q_{i}-q_{i-1} \leq \sqrt{n}$ for any $i, 1 \leq i \leq k$ (a method for obtaining the sequence $\left\{q_{i}: 0 \leq i \leq k\right\}$ is described in section 5.2.1);

- $d c[x, y, i]=$ distance from vertex $x$ to vertex $y$ in $G-[\hat{u}, \hat{v}]$, where $\hat{u}$ is the successor of $v c[x, y, i]$ in $\pi_{x y}$ and $\hat{v}=v c[x, y, i+1]$ if $h_{\hat{v} y}>\sqrt{n}$ and is undefined otherwise;

- $b c[x, y]=$ index $i$ such that $q_{i}+1 \leq h_{x y} \leq q_{i+1}$.

5.2. Preprocessing. As distances $d_{x y}$ and $h_{x y}$ are easily initialized from shortest path trees of $G$, we focus on constructing matrices $d h, d t, d c, v c$, and $b c$. Since bands $B_{T(x)}(i, i)$ contain paths formed by single vertices, which are trivially pairwise independent in $T(x)$, constructing matrix $d h$ can be done by performing calls to algorithm fast-exclude $\left(G, x, B_{T(x)}(i, i)\right)$, presented in section 3 , for each vertex $x$ and for each $i$ such that $0<i \leq \sqrt{n}$. Similarly, matrix $d t$ can be initialized via calls to algorithm fast-exclude $\left(\widehat{G}, y, B_{\widehat{T}(y)}(i, i)\right)$ for each vertex $y$ and for each $i$ such that $0<i \leq 2 \sqrt{n}$.

To compute $d c$, we consider the problem of cutting each shortest path tree $T(x)$ into at most $2 \sqrt{n}$ bands of height $\leq \sqrt{n}$, finding a suitable subset of each band containing pairwise independent shortest paths in $T(x)$, and calling fast-exclude to compute distances without those paths. To do so, we need to compute for each vertex $x$ a suitable sequence $\left\{q_{i}: 0 \leq i \leq k\right\}$.

5.2.1. Computing the $\boldsymbol{q}_{i}$ 's. For each vertex $x$ we wish to find a sequence $q_{0}=0<q_{1}<\cdots<q_{k}<n$ of length $k+1 \leq 2 \sqrt{n}$ such that $q_{i}-q_{i-1} \leq \sqrt{n}$ for any $i, 1 \leq i \leq k$, and compute a subset of paths in $B_{T(x)}\left(q_{i}+1, q_{i+1}\right)$ that are pairwise independent in $T(x)$. The following claim provides a nice combinatorial property on trees that helps us solve the problem.

Let $T$ be a rooted directed tree with $n$ vertices and let size $(v)$ be the number of vertices in the subtree of $T$ rooted at $v$. Let $T^{\prime}$ be obtained from $T$ by deleting any vertex $u$ such that $\operatorname{size}(u) \leq \sqrt{n}$ in $T$. 
Claim 6. For any directed tree $T$ with $n$ vertices, at most $\sqrt{n}$ vertices in $T^{\prime}$ have out-degree $>1$.

Proof. Since $T^{\prime}$ contains only vertices that have size greater than $\sqrt{n}$ in $T, T^{\prime}$ has at most $\sqrt{n}$ leaves. This implies that at most $\sqrt{n}$ vertices of $T^{\prime}$ have out-degree $>1$.

Let $l_{0}<l_{1}<\cdots<l_{k}$ be the sequence of levels in $T^{\prime}$ such that at each level $l_{i}$ there is a vertex with out-degree $>1$ in $T^{\prime}$ (Figure 5.1(a)). By Claim $6, k \leq \sqrt{n}$. We now observe that cutting $T^{\prime}$ at each $l_{i}$ yields bands of vertex-disjoint paths.

Claim 7. For any $i, 1 \leq i<k, B_{T^{\prime}}\left(l_{i}+1, l_{i+1}\right)$ is a band of vertex-disjoint paths.

Proof. $B_{T^{\prime}}\left(l_{i}+1, l_{i+1}\right)$ contains all paths that connect vertices at level $l_{i}+1$ with vertices at level $l_{i+1}$ in $T^{\prime}$. The proof easily follows by observing that, by the definition of sequence $\left\{l_{i}\right\}$, all vertices in $T^{\prime}$ at levels $l_{i}+1$ to $l_{i+1}-1$ have out-degree $\leq 1$.

Notice that, since by Claim $7 B_{T^{\prime}}\left(l_{i}+1, l_{i+1}\right)$ is a band of vertex-disjoint paths and all of them start at the same level $l_{i}+1$ in $T^{\prime}$, then they are clearly pairwise independent in $T^{\prime}$. As $T^{\prime}$ is obtained by pruning $T, B_{T^{\prime}}\left(l_{i}+1, l_{i+1}\right) \subseteq B_{T}\left(l_{i}+1, l_{i+1}\right)$ and paths in $B_{T^{\prime}}\left(l_{i}+1, l_{i+1}\right)$ are also pairwise independent in $T$. Unfortunately, however, we are not guaranteed that $l_{i}-l_{i-1} \leq \sqrt{n}$, as we need for constructing $d c$. However, we note that splitting a band of vertex-disjoint paths yields again bands of vertex-disjoint paths. This leads to the following claim.

Claim 8. If $B_{T}(i+1, j)$ is a band of vertex-disjoint paths, then for any $i<h<j$, both $B_{T}(i+1, h)$ and $B_{T}(h+1, j)$ are bands of vertex-disjoint paths.

Let $s_{0}<s_{1}<\cdots<s_{\lfloor\sqrt{n}\rfloor}$ be a sequence such that $s_{i}=i \cdot\lfloor\sqrt{n}\rfloor$ (Figure 5.1b). By Claim 8, if we merge sequences $\left\{l_{i}\right\}$ and $\left\{s_{i}\right\}$ and get rid of duplicates, we obtain an ordered sequence $\left\{q_{i}\right\}$ of length at most $2 \sqrt{n}$ with the desired properties (Figure 5.1c).

5.2.2. Computing $\boldsymbol{v} \boldsymbol{c}, \boldsymbol{d} \boldsymbol{c}$, and $\boldsymbol{b} \boldsymbol{c}$. We remark that $\left\{q_{i}\right\}$ induces at most $2 \sqrt{n}$ bands of vertex-disjoint paths in $T^{\prime}(x)$ with height $\leq \sqrt{n}$. Clearly, these paths are pairwise independent in $T^{\prime}(x)$. To initialize $d c$, we can thus perform calls to fast-exclude $\left(G, x, B_{T^{\prime}(x)}\left(q_{i}+1, q_{i+1}\right)\right)$ for each vertex $x$ and for each $0<i \leq 2 \sqrt{n}$. Again, we can use fast-exclude instead of exclude.

At this point, one may argue that excluding only independent paths in $T^{\prime}(x)$, which is obtained by pruning $T(x)$, might not give the correct result for some $d c[x, y, i]$ if $y \notin T^{\prime}(x)$. However, $d c[x, y, i]$ is defined only when $h_{\hat{v} y}>\sqrt{n}$, where $\hat{v}=$ $v c[x, y, i+1]$, and $\hat{v} \in T^{\prime}(x)$ in this case. Thus $d c[x, y, i]$ is correctly computed by calling fast-exclude $\left(G, x, B_{T^{\prime}(x)}\left(q_{i}+1, q_{i+1}\right)\right)$.

Finally, we observe that once sequences $\left\{q_{i}\right\}$ have been computed for each $T(x)$, matrices $v c$ and $b c$ can be easily initialized.

5.3. Query. The query algorithm is shown in Figure 5.2. We first get rid of the cases where $v \notin \pi_{x y}$ and $h_{v y} \leq 2 \sqrt{n}$, for which the answers are stored explicitly in $d_{x y}$ and $d t\left[x, y, h_{v y}\right]$, respectively (lines 1-2). In line 3 we retrieve the unique index $i$ such that $v$ falls in $B_{T(x)}\left(q_{i}+1, q_{i+1}\right)$, and then in lines $4-5$ we identify the vertices $\widehat{u}$ and $\widehat{v}$ on the path $\pi_{x y}$ in $T(x)$ that are at levels $q_{i}+1$ and $q_{i+1}$, respectively. The correct answer is given in line 6 by accessing matrices $d c, d h$, and $d t$.

5.4. Analysis. To prove the correctness of v-dist in the case that lines 3-7 are executed, we first note that $h_{v y}>2 \sqrt{n}$ implies $h_{\hat{v} y}>\sqrt{n}$, since by construction $q_{i}-q_{i-1} \leq \sqrt{n}$, and thus $d c[x, y, i]$ is well defined. We now prove that the answer takes into account all possible configurations of the endpoints of detours $\pi_{a b}^{u v}$ (see Figure 2.1). It is easy to see that $x<\widehat{u}<v<\widehat{v}<y$ and 


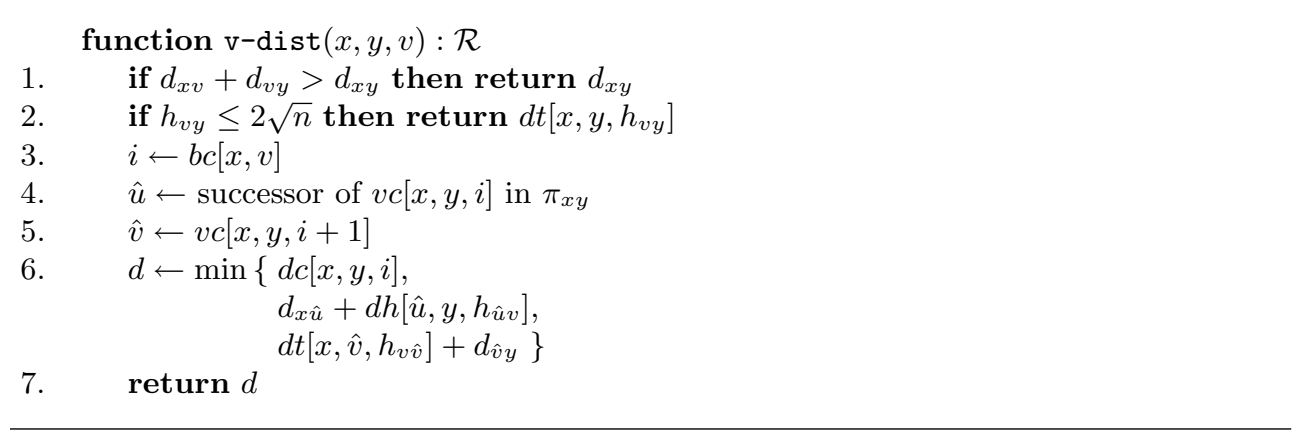

FIG. 5.2. Query algorithm for the second oracle.

- $d c[x, y, i]=d_{x y}(x, \widehat{u}, \widehat{v}, y)$,

- $d_{x \hat{u}}+d h\left[\hat{u}, y, h_{\hat{u} v}\right]=d_{x y}(\widehat{u}, v, v, y)$, and

- $d t\left[x, \hat{v}, h_{v \hat{v}}\right]+d_{\hat{v} y}=d_{x y}(x, v, v, \widehat{v})$.

Thus, by Claim $3, d=d_{x y}(x, v, v, y)$.

Claim 9. Preprocessing requires $O\left(m n^{1.5}+n^{2.5} \log n\right)$ worst-case time and any $\mathrm{v}$-dist operation requires $O(1)$ worst-case time.

Proof. Growing shortest path trees $T(x)$ for all vertices $x$ requires $O\left(m n+n^{2} \log n\right)$ time in the worst case [10]. The proof for the preprocessing follows from Claim 4 by observing that initializing $d c, d h$, and $d t$ is carried out via $O(\sqrt{n})$ calls to fast-exclude for each vertex $x$. The bound for queries is straightforward.

6. Handling edge failures. The oracles in the previous two sections can be easily extended to handle edge failures by maintaining one additional matrix de of size $n \times n$ for any $x$ and $y$ :

- $d e[x, y]=$ distance from vertex $x$ to vertex $y$ in $G$ without the first edge of $\pi_{x y}$.

Claim 10. The matrix de can be initialized in $O\left(m n+n^{2} \log n\right)$ time.

Proof. The proof directly follows from the properties of an earlier version of the algorithm fast-exclude in [7] based on the notion of edge-independent paths. However, since in this article we use the notion of vertex-independent paths instead, we present a proof of the claim based on it.

Consider a given $T(x)$, and let $v_{1}, v_{2}, \ldots, v_{k}$ be the children of $x$ in $T(x)$. We extend $T(x)$ to $T^{\prime}(x)$ and thus $G$ to $G^{\prime}$ by introducing $k$ new vertices $u_{1}, u_{2}, \ldots, u_{k}$ and for $1 \leq i \leq k$, replacing each edge $\left(x, v_{i}\right)$ in $T(x)$ by two consecutive edges $\left(x, u_{i}\right)$ and $\left(u_{i}, v_{i}\right)$. Clearly removing any vertex $u_{i}$ from $T^{\prime}(x)$ has the same effect as removing the corresponding edge $\left(x, v_{i}\right)$ from $T(x)$. Therefore, since the singlevertex paths in $B_{T^{\prime}(x)}(1,1)$ are trivially independent, we can compute $d e[x, y]$ for all $y \in V-\{x\}$ in time $O(m+n \log n)$ by calling fast-exclude $\left(G^{\prime}, x, B_{T^{\prime}(x)}(1,1)\right)$. (Note that we can perform the same computation in the same time bound without constructing $G^{\prime}$ explicitly but instead extending fast-exclude to handle this special case.) Since we need to call fast-exclude once for each $x \in V$, the total initialization time for de is $O\left(m n+n^{2} \log n\right)$.

6.1. Query. The query algorithm is shown in Figure 6.1. In line 1 of the algorithm, we get rid of the case where the failed edge is not on $\pi_{x y}$. In line $2, d_{1}$ is assigned the distance from $x$ to $y$ avoiding vertex $u$ and thus edge $(u, v)$. In line $3, d_{2}$ is assigned the distance from $x$ to $y$ that avoids the edge $(u, v)$ but passes through the 
function e-dist $(x, y, u, v): \mathcal{R}$

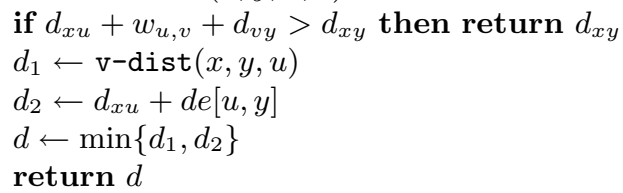

FIG. 6.1. Query algorithm for link failure.

vertex $u$. In line $4, d$ is assigned the minimum of $d_{1}$ and $d_{2}$ which is then returned in line 5 as the shortest $x$ to $y$ distance avoiding $(u, v)$.

6.2. Analysis. We observe that the paths in $G$ from $x$ to $y$ that avoid $(u, v)$ can be divided into two groups: (1) paths that avoid $u$ and (2) paths that pass through $u$. Line 2 of the algorithm finds the length of the shortest path in group (1), and line 3 does the same for group (2). Thus the minimum of the two distances obtained in lines 2 and 3 gives the required distance. Note that since $v$-dist runs in constant worst-case time, so does e-dist.

6.3. Avoiding two consecutive edges. In this section we observe that the distance from any vertex $x$ to another vertex $y$ avoiding two consecutive failed edges on $\pi_{x y}$ can be computed in constant time by maintaining another $n \times n$ matrix $\widehat{d e}$, which is the dual of de in $\widehat{G}$ :

- $\widehat{d e}[x, y]=$ distance from vertex $y$ to vertex $x$ in $\widehat{G}$ without the first edge of $\hat{\pi}_{y x}$.

Assuming that the two consecutive failed links are $(u, v)$ and $(v, w)$, all paths in $G$ from $x$ to $y$ avoiding those two edges can be divided into two groups: (1) paths that avoid $v$ (the length of the shortest such path can be found by calling $\mathrm{v}$-dist $(x, y, v)$ ) and (2) paths that pass through $v$ (the length of the shortest such path is given by $\widehat{d e}[x, v]+d e[v, y])$. Thus the smaller of these distances is the required distance.

7. Supporting path queries. The oracles presented in this paper can easily be extended to support path queries of the form $\mathrm{v}$-path $(x, y, v)$ and $\mathrm{e}-\operatorname{path}(x, y, u, v)$, which return the first edge on the shortest path from vertex $x$ to vertex $y$ in $G-\{v\}$ and in $G-\{(u, v)\}$, respectively.

In this section, we show how to extend the oracle given in section 4 to support $\mathrm{v}$-path $(x, y, v)$ queries. Extending the other oracles and $\operatorname{supporting} \mathrm{e}-\mathrm{path}(x, y, u, v)$ queries can easily be done in a similar way. We add to the data structure of section 4.1 the following additional matrices:

- $d l e[x, y, i]=\left(x, x^{\prime}\right)$, where $\left(x, x^{\prime}\right)$ is the first edge on the shortest path from vertex $x$ to vertex $y(\neq x)$ in $G-\pi$, and $\pi$ is the subpath of $\pi_{x y}$ starting at level $2^{i-1}$ and ending at level $2^{i}-1\left(1 \leq i \leq \log _{2} h_{x y}\right)$ in $T(x)$;

- $\operatorname{dre}[x, y, i]=\left(x, x^{\prime}\right)$, where $\left(x^{\prime}, x\right)$ is the last edge on the shortest path from vertex $y$ to vertex $x(\neq y)$ in $\widehat{G}-\pi$, and $\pi$ is the subpath of $\widehat{\pi}_{y x}$ starting at level $2^{i-1}$ and ending at level $2^{i}-1\left(1 \leq i \leq \log _{2} h_{x y}\right)$ in $\widehat{T}(y)$;

- $\operatorname{sle}[x, y, i]=\left(x, x^{\prime}\right)$, where $\left(x, x^{\prime}\right)$ is the first edge on the shortest path from vertex $x$ to vertex $y(\neq x)$ in $G-\{v\}$, and $v$ is the vertex of $\pi_{x y}$ at level $2^{i-1}$ $\left(1 \leq i<1+\log _{2} h_{x y}\right)$ in $T(x)$

- $\operatorname{sre}[x, y, i]=\left(x, x^{\prime}\right)$, where $\left(x^{\prime}, x\right)$ is the last edge on the shortest path from 


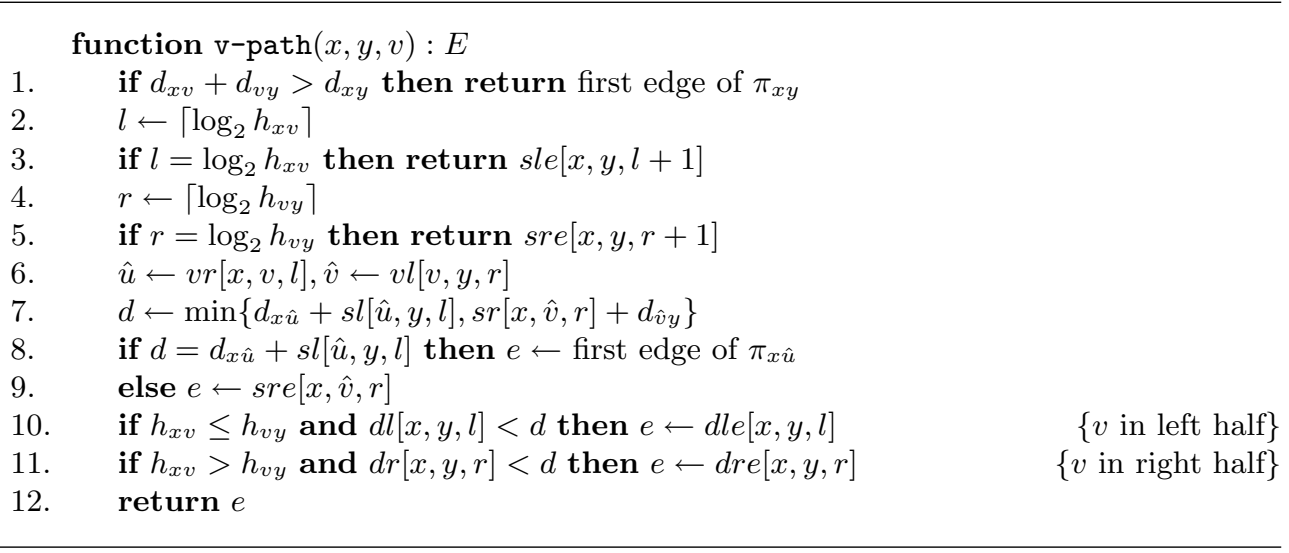

FIG. 7.1. Path version of the query algorithm for our first oracle.

vertex $y$ to vertex $x(\neq y)$ in $\widehat{G}-\{v\}$, and $v$ is the vertex of $\widehat{\pi}_{y x}$ at level $2^{i-1}$ $\left(1 \leq i<1+\log _{2} h_{x y}\right)$ in $\widehat{T}(y)$.

Matrices dle, dre, sle, and sre can easily be initialized in the preprocessing phase within the same time bounds by a simple extension of procedures exclude and fast-exclude described in section 3. Figure 7.1 shows an implementation of operation $\mathrm{v}$-path obtained as a modification of the query procedure v-dist given Figure 4.1. The analysis is straightforward and is left to the reader.

8. A space lower bound. In this section, we briefly discuss a space lower bound for the single-source version of the distance sensitivity problem. This version is relevant for shortest path routing in networks [16]: a router cares only about itself as a source when deciding which outgoing link to use when forwarding a packet on a shortest path to its destination. We would therefore like a single-source routing table working under each possible failure. The solution in [24] uses $O(h n)$ space if $h$ is the maximal hop count on a shortest path to a destination. However, we might have $h=\Omega(n)$. Corresponding to our $O\left(n^{2} \log n\right)$ space solution for the all pairs case, we would like an $O(n \log n)$ space solution for the single-source case. For single-source and single-destination we can get down to $O(n)$ space if the graph is undirected, and this includes a representation of the alternative paths [11, 19], but now we want an $\tilde{O}(n)$ solution working for all possible destinations and failures. Below we show that this is impossible if $m$ is large. In fact, we will prove that the $O(h \cdot n)$ space bound from [24] is tight. More precisely, for any number of vertices $n>2$, and $h<n$, we will demonstrate a graph with $m=\Theta(h n)$ edges, with maximal hop count $h$ in shortest paths from a specified source, and so that any single-source distance oracle for failures requires $\Omega(h n)$ space no matter whether it is for edge or vertex failures or for directed or undirected graphs.

First we present the construction for $h=n-1$. We assume that the word length $w$ is even and at least $2(1+\log n)$. Let the vertices be $v_{0}, \ldots, v_{n-1}$ where $v_{0}$ is the source vertex. For each $i<j$, we have an edge $\left(v_{i}, v_{j}\right)$, and hence a total of $\left(\begin{array}{l}n \\ 2\end{array}\right)$ edges. Each edge will have an arbitrary half word stored in the least significant bits of its weight. From the answers of a failure distance oracle, we will be able to recover all these half words. It then follows that the full representation of a failure distance oracle requires at least $\left(\begin{array}{l}n \\ 2\end{array}\right) / 2$ words. 
The low part of a weight represents numbers below $2^{w / 2}$, and the high part represents multiples of $2^{w / 2}$. Hence, if the low part is $x_{0}$ and the large part is $x_{1}$, the weight represented is $x_{0}+2^{w / 2} x_{1}$. If we know the full weight, we can easily recover the low half word with the stored information.

We will now describe how to fill the high parts of the weights. The edges $\left(v_{i}, v_{i+1}\right)$ in the path $\left(v_{0}, v_{1}, \ldots, v_{n-1}\right)$ are all given a high part of 0 . The shortest path tree will consist of this path. For an edge $\left(v_{i}, v_{j}\right)$ with $j \geq i+2$, the high part is $2 n-j+i$. It is easy to see that if link $\left(v_{i}, v_{i+1}\right)$ or vertex $v_{i+1}$ fails and we want to go to $v_{j}$, $j \geq i+2$, then the unique shortest path first follows the original shortest path from $v_{0}$ to $v_{i}$ and then switches to the link $\left(v_{i}, v_{j}\right)$. All this is true in both the directed and the undirected cases.

With the above setting of the high parts of the weights, we can first use the regular distances to find the weights along the path; that is, the weight of $\left(v_{i}, v_{i+1}\right)$ is the distance to $v_{i+1}$ minus that to $v_{i}$. Next, for each $i$ and $j \geq i+2$, we fail $\left(v_{i}, v_{i+1}\right)$ to get a distance to $v_{j}$, which is the known weight of the path $\left(v_{0}, \ldots, v_{i}\right)$ plus the weight of $\left(v_{i}, v_{j}\right)$. Thus, if we have a distance oracle that can handle failures, then we can recover all the weights and hence all the low parts with arbitrary stored information.

In the case where $h<n-1$, we start with the source vertex $v_{0}$, and then we create $\lceil n-1 / h\rceil$ paths from $v_{0}$, each of length between $h / 2$ and $h$. Thus, if we removed $v_{0}$, the graph would fall into a set of disjoint paths. We now apply the previous construction to each of the paths from $v_{0}$. If a path has length $h^{\prime}$, it uses $\left(\begin{array}{c}h^{\prime} \\ 2\end{array}\right)=\Theta\left(h^{2}\right)$ edges. Thus we get a total of $\Theta(n h)$ edges, all of whose weights can be recovered by a failure distance oracle. The representation of the distance oracle therefore requires a space of $\Theta(n h)$ words.

9. Conclusions. We have presented compact data structures for maintaining information about shortest paths in a weighted directed graph in cases of both vertex failures and edge failures. We have shown that, surprisingly, such a data structure can be stored using nearly the same space required to store a single distance matrix, while still supporting queries in constant time. Our oracle can easily be constructed in $O\left(m n^{2}+n^{3} \log n\right)$ time, matching the preprocessing time of all pairs variants of similar problems such as most vital node detection [18] and Vickrey pricing [11] in networks; while these algorithms require $\Theta\left(n^{3}\right)$ space, our oracle requires only $O\left(n^{2} \log n\right)$ space. Furthermore, we have shown that by using $O\left(n^{2.5}\right)$ space we can improve construction time to $O\left(m n^{1.5}+n^{2.5} \log n\right)$.

Our oracles are different from the case of dynamic algorithms, where distances have to be updated after each vertex or edge failure. Instead, our oracles are already prepared to answer distance queries following the failure of any single vertex or edge, and so the delay time in answering a query is minimized. If failures in a network happen quite rarely, when a node or link goes down we have time to construct a new oracle in the background to cope with a possible additional failure. It would be interesting to explore whether compact oracles with fast query time that are able to deal with more than one failure at a time can be constructed. Finally, can we further improve construction time?

\section{REFERENCES}

[1] M. O. Ball, B. L. Golden, And R. V. Vohra, Finding the most vital arcs in a network, Oper. Res. Lett., 8 (1989), pp. 73-76. 
[2] A. Bar-Noy, S. Khuller, And B. Schieber, The Complexity of Finding Most Vital Arcs and Nodes, Technical report CS-TR-3539, Institute for Advanced Studies, University of Maryland, College Park, MD, 1995.

[3] R. A. Chowdhury and V. Ramachandran, Improved distance oracles for avoiding a linkfailure, in Proceedings of the 13th International Symposium on Algorithms and Computation (ISAAC'02), Vancouver, Canada, Lecture Notes in Comput. Sci. 2518, Springer-Verlag, New York, 2002, pp. 523-534.

[4] H. W. Corley And D. Y. Sha, Most vital links and nodes in weighted networks, Oper. Res. Lett., 8 (1982), pp. 157-160.

[5] C. Demetrescu AND G. F. Italiano, Fully dynamic all pairs shortest paths with real edge weights, in Proceedings of the 42nd IEEE Annual Symposium on Foundations of Computer Science (FOCS'01), Las Vegas, NV, 2001, pp. 260-267.

[6] C. Demetrescu and G. F. Italiano, A new approach to dynamic all pairs shortest paths, J. ACM, 51 (2004), pp. 968-992.

[7] C. Demetrescu And M. Thorup, Oracles for distances avoiding a link-failure, in Proceedings of the 13th Annual ACM-SIAM Symposium on Discrete Algorithms (SODA'02) (San Francisco, CA), SIAM, Philadelphia, ACM, New York, 2002, pp. 838-843.

[8] E. W. Dijkstra, A note on two problems in connection with graphs, Numer. Math., 1 (1959), pp. 269-271.

[9] B. Fortz And M. Thorup, Internet traffic engineering by optimizing OSPF weights, in Proceedings of the 19th IEEE INFOCOM, The Conference on Computer Communications, Tel-Aviv, Israel, 2000, pp. 519-528.

[10] M. L. Fredman And R. E. TARJAn, Fibonacci heaps and their use in improved network optimization algorithms, J. ACM, 34 (1987), pp. 596-615.

[11] J. Hershberger and S. Suri, Vickrey prices and shortest paths: What is an edge worth?, in Proceedings of the 42nd IEEE Annual Symposium on Foundations of Computer Science (FOCS'01), Las Vegas, NV, 2001, pp. 252-259. Erratum in FOCS'02.

[12] J. Hershberger, S. Suri, And A. Bhosle, On the difficulty of some shortest path problems, in Proceedings of the 20th International Symposium of Theoretical Aspects of Computer Science (STACS'03), Berlin, Germany, 2003, pp. 343-354.

[13] V. KING, Fully dynamic algorithms for maintaining all-pairs shortest paths and transitive closure in digraphs, in Proceedings of the 40th IEEE Symposium on Foundations of Computer Science (FOCS'99), New York, 1999, pp. 81-99.

[14] V. King And G. SAgert, A fully dynamic algorithm for maintaining the transitive closure, J. Comput. System Sci., 65 (2002), pp. 150-167.

[15] V. King And M. Thorup, A space saving trick for directed dynamic transitive closure and shortest path algorithms, in Proceedings of the 7th Annual International Computing and Combinatorics Conference (COCOON'01), Guilin, China, Lecture Notes in Comput. Sci. 2108, Springer-Verlag, New York, 2001, pp. 268-277.

[16] J. Moy, OSPF: Anatomy of an Internet Routing Protocol, Addison-Wesley, Reading, MA, 1999.

[17] A. Mas-Collel, W. Whinston, and J. Green, Microeconomic Theory, Oxford University Press, Oxford, UK, 1999.

[18] E. Nardelli, G. Proietti, And P. Widmayer, Finding the most vital node of a shortest path, in Proceedings of the 7th Annual International Computing and Combinatorics Conference (COCOON'01), Guilin, China, Lecture Notes in Comput. Sci. 2108, Springer-Verlag, New York, 2001, pp. 278-287.

[19] N. Nisan and A. Ronen, Algorithmic mechanism design, in Proceedings of the 31st Annual ACM Symposium on Theory of Computation (STOC'99), ACM, New York, 1999, pp. $129-140$

[20] S. Pettie, A new approach to all-pairs shortest paths on real-weighted graphs, Theoret. Comput. Sci., 312 (2003), pp. 47-74.

[21] L. RODITTY AND U. ZWICK, Replacement paths and $k$ simple shortest paths in unweighted directed graphs, in Proceedings of the 32nd International Colloquium on Automata, Languages, and Programming (ICALP'05), Lisboa, Portugal, Lecture Notes in Comput. Sci. 3580, Springer-Verlag, New York, 2005, pp. 249-260.

[22] T. TAKAOKA, Subcubic cost algorithms for the all pairs shortest path problem, Algorithmica, 30 (1998), pp. 309-318.

[23] M. ThORUP, Undirected single source shortest paths with positive integer weights in linear time, J. ACM, 46 (1999), pp. 362-394.

[24] M. ThORUP, Fortifying OSPF/IS-IS Against Link-Failure, manuscript, 2001.

Copyright (c) by SIAM. Unauthorized reproduction of this article is prohibited. 
[25] M. Thorup, Fully dynamic all-pairs shortest paths: Faster and allowing negative cycles, in Proceedings of the 9th Scandinavian Workshop on Algorithm Theory (SWAT'04), Humlebaek, Denmark, 2004, pp. 384-396.

[26] M. Thonup, Integer priority queues with decrease key in constant time and the single source shortest paths problem, J. Comput. System Sci., 69 (2004), pp. 330-353.

[27] U. ZWICK, A slightly improved sub-cubic algorithm for the all pairs shortest paths problem with real edge lengths, in Proceedings of the 15th International Symposium on Algorithms and Computation (ISAAC'04), Hong Kong, China, Lecture Notes in Comput. Sci. 3341, Springer-Verlag, 2004, pp. 921-932.

Copyright (c) by SIAM. Unauthorized reproduction of this article is prohibited. 